\title{
Estado nutricional do pessegueiro na principal região produtora de pêssego para indústria no Brasil
}

\author{
Nutritional status of peach trees in Brazil's main commercial peach-growing region \\ Renan Navroski ${ }^{1 *}$, Jorge Atílio Benati ${ }^{1}$, Caroline Farias Barreto ${ }^{2}$, Lucas De Oliveira Fischer ${ }^{1}$, José \\ Francisco Martins Pereira ${ }^{2}$, Gilberto Nava ${ }^{2}$, Marcelo Barbosa Malgarim ${ }^{1}$ \\ ${ }^{1}$ Universidade Federal de Pelotas, Pelotas, RS, Brasil. *Autor para correspondência: navroski@outlook.com \\ ${ }^{2}$ Embrapa Clima Temperado, Pelotas, RS, Brasil.
}

Submissão:01/06/2020 / Aceite: 05/09/2020

\begin{abstract}
RESUMO
O presente trabalho teve como objetivo caracterizar nutricionalmente plantas de pessegueiro na região produtora de Pelotas, RS. Foram amostrados 65 pomares de pessegueiros das cultivares Maciel, Granada, Esmeralda e Sensação nos municípios de Pelotas, Canguçu e Morro Redondo no ciclo produtivo de 2018. As amostras foliares, após secas e moídas, foram analisadas quanto aos seus teores de nitrogênio $(\mathrm{N})$, fósforo $(\mathrm{P})$, potássio $(\mathrm{K})$, cálcio $(\mathrm{Ca})$, magnésio $(\mathrm{Mg})$, boro $(\mathrm{B})$, cobre $(\mathrm{Cu})$, ferro $(\mathrm{Fe})$, manganês $(\mathrm{Mn})$ e zinco $(\mathrm{Zn})$ e os resultados foram apresentados em distribuições de frequência nas classes de suficiência indicadas para a cultura na região além da correlação entre os nutrientes. Observou-se maiores deficiências nos teores de nitrogênio, cobre, ferro e zinco. Entretanto, para a maioria das amostras, os teores de potássio encontraram-se na classe "acima do normal", encontrou-se correlações entre $\mathrm{P} / \mathrm{Ca}, \mathrm{P} / \mathrm{Cu}, \mathrm{Ca} / \mathrm{P}, \mathrm{Ca} / \mathrm{Mg}$ e $\mathrm{Ca} / \mathrm{B}$. É possível constatar a carência do uso de ferramentas para avaliar o estado nutricional dos pomares, principalmente a diagnose foliar, o que levou ao uso inadequado de fertilizantes e consequentemente um desbalanço nutricional das plantas.
\end{abstract}

PALAVRAS-CHAVE: adubação, análise foliar, diagnose nutricional, Prunus persica.

\begin{abstract}
This study set out to assess the nutritional status of peach trees in the commercial peach-growing region of Pelotas, Rio Grande do Sul. The sample includes 65 peach orchards of the Maciel, Granada, Esmeralda, and Sensação cultivars in the municipalities of Pelotas, Canguçu, and Morro Redondo during the 2018 agricultural cycle. After drying and grinding the leaf samples for an analysis of their nitrogen $(\mathrm{N})$, phosphorus $(\mathrm{P})$, potassium $(\mathrm{K})$, calcium $(\mathrm{Ca})$, magnesium $(\mathrm{Mg})$, boron $(\mathrm{B})$, copper $(\mathrm{Cu})$, iron $(\mathrm{Fe})$, manganese $(\mathrm{Mn})$, and zinc $(\mathrm{Zn})$ content, the results were presented in frequency distributions divided into classes based on the nutrient requirements indicated for the crop in the region, in addition to the correlations between the nutrients. Greater deficiencies were observed in nitrogen, copper, iron, and zinc content. However, potassium content was found in the "above normal" class for most of the samples, and there were correlations between $\mathrm{P} / \mathrm{Ca}, \mathrm{P} / \mathrm{Cu}, \mathrm{Ca} / \mathrm{P}, \mathrm{Ca} / \mathrm{Mg}$, and $\mathrm{Ca} / \mathrm{B}$. An insufficient use of the tools to assess the nutritional status of the orchards was observed, especially the leaf diagnosis, which led to the inappropriate use of fertilizers and the consequent nutrient imbalance in the plants.
\end{abstract}

KEYWORDS: fertilization, leaf analysis, nutrition diagnosis, Prunus persica.

\section{INTRODUÇÃO}

O estado do Rio Grande do Sul possui $74,6 \%$ da área plantada de pessegueiro do Brasil e responde por $66,7 \%$ da produção nacional desta fruta (IBGE 2018). A região sul do Rio Grande do Sul, mais especificamente os municípios de Pelotas, Canguçu e Morro Redondo, respondem por $34 \%$ da área plantada de pessegueiro no Brasil (MAYER et al. 2019). A maioria da produção destes três municípios é voltada para a indústria de compotas (pêssego em calda), que representa cerca de $90 \%$ da produção de compotas do estado do Rio Grande do Sul e por, aproximadamente, $58 \%$ da produção nacional de pêssego em calda, estimando-se que o pêssego seja responsável, na microrregião de Pelotas, pela geração de aproximadamente 2,5 mil empregos diretos (SEVERO 2017). 
Entretanto, esta região se caracteriza por baixas produtividades, sendo a menor dentre os seis principais estados produtores brasileiros (MAYER et al. 2016). A baixa produtividade pode estar relacionada a alguns fatores, como clima, solo, infraestrutura no meio rural e técnicas de manejo inadequadas.

Dentre esses fatores, a adubação é uma das principais práticas agronômicas na cultura do pessegueiro que determinam a produtividade da cultura (FREIRE \& MAGNANI 2014). A heterogeneidade das características edafoclimáticas, aliada a falta de acompanhamento do estado nutricional das plantas, faz com que, muitas vezes, a prática da adubação seja feita de forma empírica ou por especulação agronômica, ou seja, sem uso de ferramentas como análise de solo ou foliar, levando o produtor a fazer adubação muitas vezes inadequada (FREIRE \& MAGNANI 2014).

Sendo assim, é necessário o desenvolvimento de pesquisas com o intuito de melhorar as técnicas de cultivo, e assim, elevar a produtividade dos pomares e a renda dos produtores desta região (NAVROSKI et al. 2019a). Alguns trabalhos têm sido realizados sobre adubação dos pessegueiros nos últimos anos (FERREIRA et al. 2018, NAVROSKI et al. 2019a), com finalidade de adequar as doses dos fertilizantes para aumentar a produtividade dos pomares e ou fazer um uso racional de fertilizantes, proporcionando uma redução de custos.

Deste modo, o presente trabalho teve como objetivo levantar e caracterizar a condição nutricional da cultura do pessegueiro na região produtora de Pelotas através do diagnóstico foliar a fim de identificar possíveis desbalanços nutricionais responsáveis pela baixa produtividade da cultura.

\section{MATERIAL E MÉTODOS}

O presente trabalho foi realizado no ciclo produtivo de 2018, na região produtora de Pelotas, RS (Pelotas, Canguçu e Morro Redondo) em 65 pomares de pessegueiros das cultivares Maciel, Granada, Sensação e Esmeralda, que segundo MAYER et al. (2019) estão entre as mais cultivadas com a finalidade de industrialização. Os dados climáticos da região estão apresentados na Figura 1, retirados do Posto

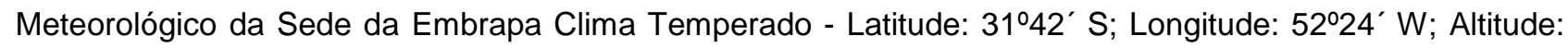
$57 \mathrm{~m}$. Segundo a classificação Köppen, o clima da região é do tipo Cfa com verões quentes (ALVARES et al. 2013).

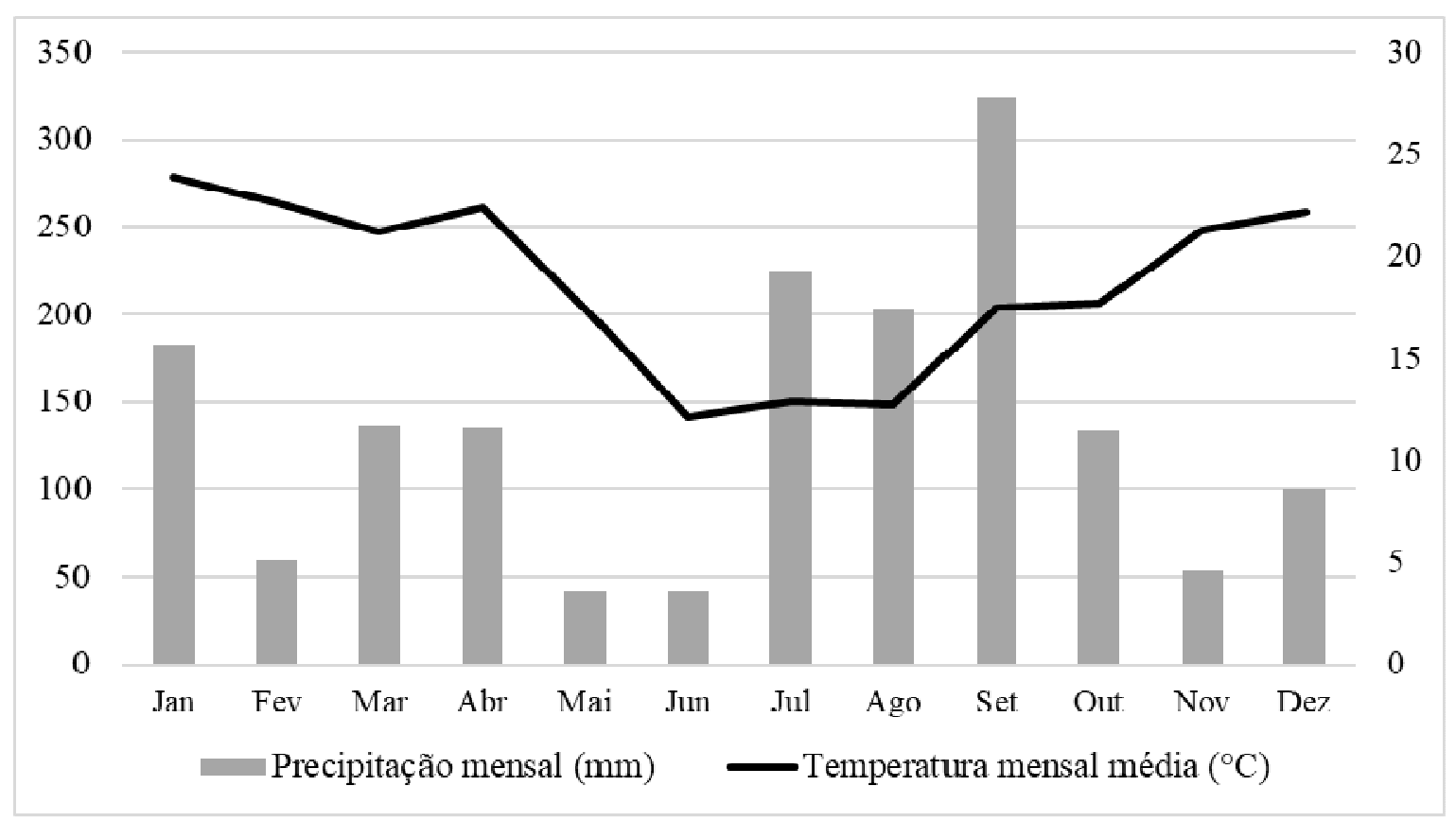

Figura 1. Precipitação mensal $(\mathrm{mm})$ e temperatura mensal média $\left({ }^{\circ} \mathrm{C}\right)$ no ano de 2018 na região de Pelotas, RS. (AGROMET 2018).

Figure 1. Monthly precipitation $(\mathrm{mm})$ and average monthly temperature $\left({ }^{\circ} \mathrm{C}\right)$ in 2018 in the region of Pelotas, RS. (AGROMET 2018).

Nestes pomares foram realizadas a amostragem de folhas para análises químicas de tecido vegetal. As coletas foram amostradas na época padrão indicada pela CQFS-RS/SC (2016), entre a $13^{\text {a }}$ e a $15^{\text {a }}$ semana após a plena floração, respeitando o ciclo de cada cultivar, coletando-se folhas de nove plantas para se obter uma amostra composta de tecido foliar, totalizando 65 amostras. 
As amostras foram secas em estufa a $65^{\circ} \mathrm{C}$ até peso constante, moídas em moinho de amostras da marca IKA, modelo A11 e analisadas quanto aos teores minerais conforme metodologias descritas por CARMO et al. (2000). Para o N utilizou-se o analisador elementar TruSpec CHN-S. Para P, K, Ca, Mg, Cu, $\mathrm{Zn}, \mathrm{Mn}$ e Fe realizou-se a digestão nítrico-perclória para obtenção do extrato. O $\mathrm{P}$ foi analisado por espectrometria com amarelo de vanadato. $\mathrm{K}$ foi determinado em fotômetro de chama marca Digmed DM-62. $\mathrm{Ca}, \mathrm{Mg}, \mathrm{Cu}, \mathrm{Zn}$ e $\mathrm{Mn}$ determinados em espectrofotômetro de absorção atômica marca Perkin Elmer AAnalyst 200. O boro (B) foi solubilizado por digestão seca (mufla) e quantificado pelo método da Azometina-H.

Os resultados foram classificados de acordo com os teores de cada nutriente segundo classificação do CQFS-RS/SC (2016) em "insuficiente", "abaixo do normal", "normal", "acima do normal" e "excessivo" (Tabela 1), sendo feita a distribuição de frequência em cada classe por nutriente. Realizou-se também a análise de correlação linear de Pearson entre os nutrientes, para a interpretação da correlação usou-se a classificação proposta por SHIKAMURA (2006) que classifica os valores de correlação por faixas, sendo: até 0,19 correlação bem fraca; fraca de 0,20 a 0,39 ; moderada de 0,40 a 0,69 ; forte de 0,70 a 0,89 ; e muito forte, de 0,90 a 1,00 .

Tabela 1. Classificação dos teores foliares de macro e micronutrientes em pessegueiros. Adaptado de CQFS-RS/SC (2016).

Table 1. Classification of the macro and micronutrient content in peach tree leaves. Adapted from CQFSRS/SC (2016).

\begin{tabular}{lccccc}
\hline \multirow{2}{*}{ Interpretação } & $\mathrm{N}$ & $\mathrm{P}$ & $\mathrm{K}$ & $\mathrm{Ca}$ & $\mathrm{Mg}$ \\
\cline { 2 - 5 } & $<2,00$ & $<0,05$ & $<0,50$ & $<0,65$ & $<0,20$ \\
Insuficiente & $2,00-3,29$ & $0,05-0,14$ & $0,50-1,39$ & $0,65-1,69$ & $0,20-0,49$ \\
Abaixo do normal & $3,30-4,50$ & $0,15-0,30$ & $1,40-2,00$ & $1,70-2,60$ & $0,50-0,80$ \\
Normal & $4,51-6,00$ & $0,31-0,40$ & $2,01-2,80$ & $2,61-3,6$ & $0,81-1,20$ \\
Acima do normal & $>6,00$ & $>0,40$ & $>2,80$ & $>3,60$ & $>1,20$ \\
Excessivo & \multicolumn{5}{c}{ Micronutrientes $\left(\mathrm{mg} \mathrm{k}^{-1}\right)$} \\
\hline & $\mathrm{B}$ & $\mathrm{Cu}$ & $\mathrm{Mn}$ & $\mathrm{Fe}$ & $\mathrm{Zn}$ \\
\hline Insuficiente & $<3$ & - & $<20$ & $<50$ & $<10$ \\
Abaixo do normal & $3-29$ & $<6$ & $20-29$ & $50-99$ & $10-23$ \\
Normal & $30-60$ & $6-30$ & $30-160$ & $100-230$ & $24-37$ \\
Acima do normal & $61-90$ & $31-50$ & $161-400$ & $231-330$ & $38-50$ \\
Excessivo & $>90$ & $>50$ & $>400$ & $>330$ & $>50$ \\
\hline
\end{tabular}

\section{RESULTADOS E DISCUSSÃO}

Na Figura 2A estão apresentadas as distribuições de frequência dos macronutrientes onde é possível se observar que, para o nitrogênio, $93,6 \%$ das amostras foram classificadas como "abaixo do normal", 1,6\% como "insuficiente" e apenas 4,8\% como "normal". Segundo ROMBOLÀ et al. (2012) o N é o elemento de maior importância nas drupáceas. É possível que, em função de alguns solos da região serem arenosos e com baixos teores de matéria orgânica, as doses aplicadas pelos produtores sejam insuficientes para suprir as necessidades das plantas. Outro fator importante a ser considerado é que os valores de referência podem estar inadequados para a cultura na região. Em outras regiões produtoras do mundo, JOHNSON (2008) classifica teores entre 2,6 e 3,5\% como a faixa de suficiência de N, JOHNSON \& URIU (1989) citam valores entre 2,6 e $3,0 \%$, SHEAR \& FAUST (1980) valores entre 2,5 e 4,0\%, e TAYLOR (2009), valores entre 2,75 e 3,5\%. Em todos os casos, a faixa adequada está abaixo da utilizada na região de Pelotas, RS, conforme o CQFS-RS/SC (2016). O valor médio entre todas as amostras foi de 2,74\% (Tabela 2), fazendo com a classificação média da região ficasse como abaixo do normal, sendo o único macronutriente nesta classe, reforçando a hipótese da inadequação dos valores de referência utilizados na região.

Em todas as amostras analisadas os teores de P classificaram-se como "normal" ou "acima do normal" (Figura 2A), evidenciando que este nutriente é pouco limitante na cultura. Um dos principais fatores que levam a isto é a frequente utilização, por parte dos produtores, de adubos formulados (NPK) com altos teores de $\mathrm{P}$ na formulação aliado à sua aplicação consecutiva (NAVROSKI et al. 2019b), faz com que os níveis deste elemento no solo, em geral, sejam altos. Segundo NAVA \& CARVALHO (2019), quando se realiza uma correção dos teores de $\mathrm{P}$ no solo em pré-plantio, não há a necessidade do uso de fórmulas que 
contenham este nutriente, ou ainda, ROMBOLÀ et al. (2012) recomendam aplicar cerca de 40-50 kg P ha-1 a cada 3-4 anos quando seus teores estiverem adequados no solo, sendo isto, suficiente para compensar as exportações. O teor médio da região ficou em 0,27\%, classificado como normal (Tabela 2).

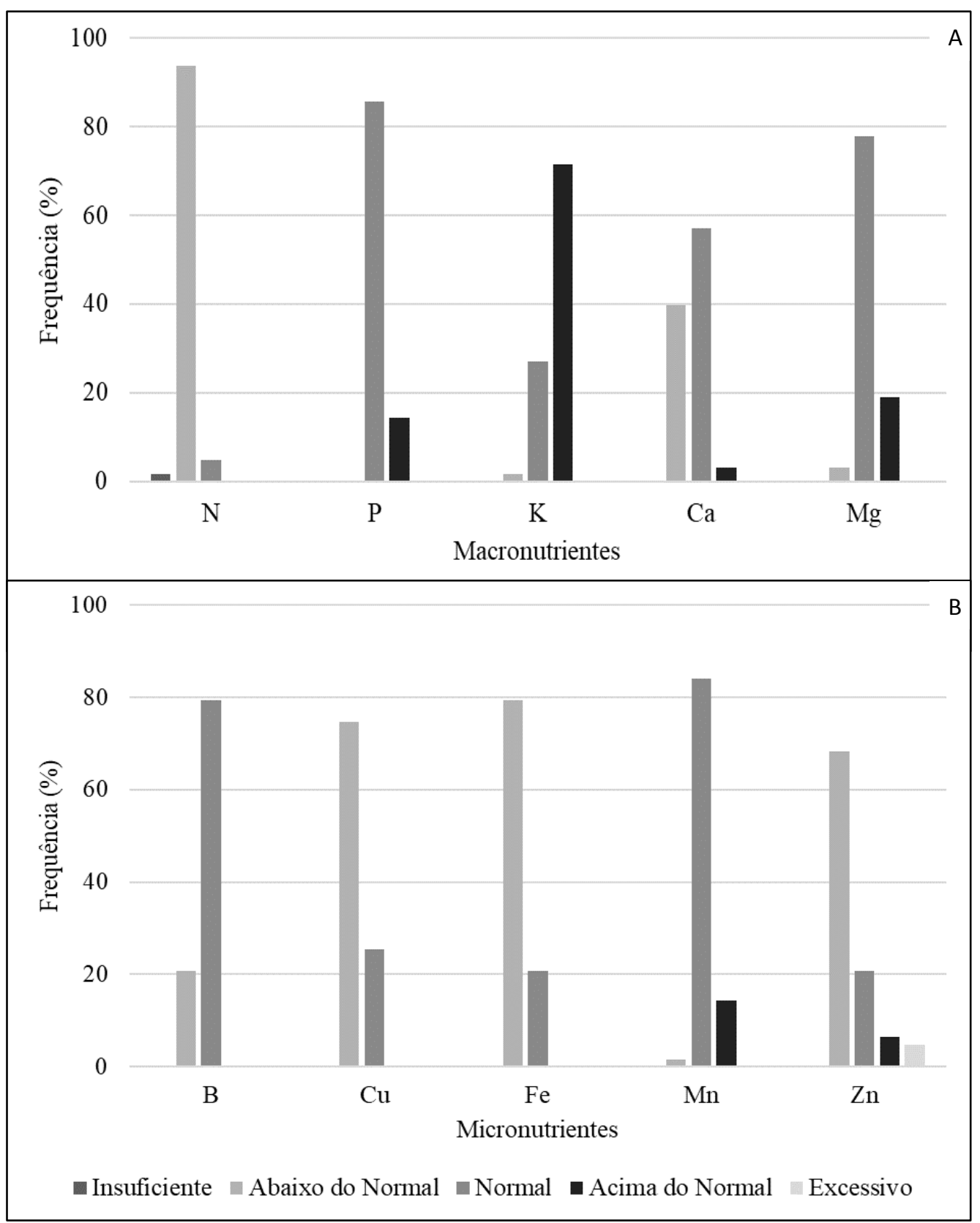

Figura 2. Distribuição de frequência dos teores de macronutrientes $(A)$ e micronutrientes $(B)$ em folhas de pessegueiros na região produtora de Pelotas, RS nas classes de suficiência adaptadas da CQFSRS/SC (2016).

Figure 2. Frequency distribution of macronutrient $(A)$ and micronutrient $(B)$ levels in peach tree leaves in the commercial peach-growing region of Pelotas, $R S$, divided into classes based on nutrient requirements, adapted from CQFS-RS/SC (2016).

O K foi o único nutriente em que a maioria das amostras $(71,4 \%)$ foi classificada como "acima do normal", revelando que pode estar havendo um uso excessivo de fertilizantes potássicos (Figura 2A). Novamente, vale citar o uso de formulações NPK prontas que são inadequadas para a cultura, causando 0 uso excessivo de $\mathrm{P}$ e $\mathrm{K}$ e podendo levar a um desbalanço nutricional. Outra hipótese é que as classes utilizadas na região do experimento estejam subestimadas, em comparação com outras regiões, como é o caso citado por JOHNSON (2008), que classifica a faixa de suficiência de K entre 2,0 e 3,0\%, sendo esta faixa considerada "acima do normal" pelo critério da CQFS-RS/SC (2016). A classificação média entre todas as amostras foi acima do normal, com média de 2,07\% (Tabela 2), o que seria considerado normal segundo JOHNSON (2008). 
Tabela 2. Teores médios, coeficiente de variação e classificação média dos teores foliares de macro e micronutrientes em pessegueiros.

Table 2. Mean nutrient levels, coefficient of variation, and mean classification for the macro and micronutrient content in peach tree leaves.

\begin{tabular}{lcccccccccc}
\hline Nutriente & $\mathrm{N}$ & $\mathrm{P}$ & $\mathrm{K}$ & $\mathrm{Ca}$ & $\mathrm{Mg}$ & $\mathrm{B}$ & $\mathrm{Cu}$ & $\mathrm{Fe}$ & $\mathrm{Mn}$ & $\mathrm{Zn}$ \\
\hline & \multicolumn{1}{c}{$\%$} & $\%$ & & & & & $\mathrm{mg} \mathrm{kg}^{-1}$ & & \\
\hline Média & 2,74 & 0,27 & 2,07 & 1,86 & 0,71 & 34,32 & 4,91 & 88,50 & 100,49 & 24,78 \\
CV (\%) & 11,7 & 11,1 & 11,2 & 19,5 & 13,3 & 15,5 & 31,1 & 34,6 & 50,8 & 52,5 \\
Classificação & $\mathrm{AbN}$ & $\mathrm{N}$ & $\mathrm{AcN}$ & $\mathrm{N}$ & $\mathrm{N}$ & $\mathrm{N}$ & $\mathrm{AbN}$ & $\mathrm{AbN}$ & $\mathrm{N}$ & $\mathrm{N}$ \\
\hline
\end{tabular}

$\overline{\mathrm{AbN}}=$ Abaixo do Normal; $\mathrm{N}=$ Normal; $\mathrm{AcN}=$ Acima do Normal. Classificação adaptada de CQFS-RS/SC (2016).

Para o $\mathrm{Ca}, 40 \%$ das amostras estavam "abaixo do normal" (Figura 2A). O Ca tem funções bem conhecidas como mensageiro, participando das relações hídricas e constituinte da parede celular (HOCKING et al. 2016). A deficiência de Ca normalmente aparece no sistema radicular e em ocasiões mais severas leva a redução do tamanho e qualidade dos frutos (JOHNSON 2008), deste modo é imprescindível o acompanhamento do estado nutricional do pomar, considerando que as deficiências nutricionais nem sempre se manifestam em órgãos da parte aérea.

Foram constatadas apenas duas amostras com teores "abaixo do normal" de Mg. A maioria das amostras $(77,7 \%)$ teve seus teores classificados como "normal". Considerando sua baixa exportação, cerca de $100 \mathrm{~g} \mathrm{t}^{-1}$ de fruto (TAGLIAVINI et al. 2000), e a disponibilidade de calcário dolomítico na região, o Mg é um nutriente pouco preocupante na cultura do pessegueiro na região produtora de Pelotas, RS.

O B teve cerca de $80 \%$ das amostras classificadas como "normal" e o restante como "abaixo do normal" (Figura 2B), demonstrando que alguns pomares necessitam de suplementação com B, entretanto, JOHNSON (2008) cita que excessos de B são muito mais prejudiciais a cultura do que deficiências, tendo em vista a facilidade de realizar sua suplementação e em função de sua mobilidade na planta. Por se tratar de um estudo em uma área de três municípios, vários fatores podem estar associados aos baixos teores de $B$, dentre eles o cultivo em solos pobres em matéria orgânica.

$\mathrm{Cu}$ e Fe apresentaram distribuições similares, em que mais de $70 \%$ das amostras foram classificadas como "abaixo do normal" (Figura 2B). O Cu, apesar de apresentar níveis baixos, pode facilmente ser corrigido, uma vez que muitos produtos fitossanitários possuem este elemento na sua composição, além disso, as práticas de manejo de inverno na cultura podem ajudar a melhorar os níveis de Cu, como por exemplo, o uso de calda bordalesa. Os pessegueiros são considerados mais suscetíveis do que a maioria das outras espécies frutíferas a deficiência de Fe (JOHNSON 2008), entretanto, as deficiências de Fe estão mais restritas a regiões com solos alcalinos. O que não é o caso desta região, de acordo com um levantamento realizado por MAYER et al. (2015), os solos da região produtora de Pelotas apresentaram pH em água abaixo de 5,4 em $68,5 \%$ dos pomares amostrados. Estes dois nutrientes tiveram suas médias entre todas as amostras classificadas como abaixo do normal (Tabela 2), sendo os dois únicos micronutrientes dentro desta classe.

Os teores de Mn classificaram-se, em sua maioria, como "normal" (Figura 2B), o Mn é considerado um nutriente pouco preocupante e geralmente, mesmo em níveis baixos, ele não afeta os componentes do rendimento em pessegueiro (JOHNSON 2008).

O Zn apresentou 68\% das amostras na classe "abaixo do normal" (Figura 2B). O Zn participa da constituição de diversas proteínas, além de fazer parte do processo de formação da auxina, o que consequentemente causa um encurtamento dos entrenós, ou seja, um rosetamento dos ramos (JOHNSON 2008). Uma causa dos baixos níveis de $\mathrm{Zn}$ foliar pode ser o alto suprimento de $P$ fornecido nas formulações aplicadas sem análises de solo ou folha, tendo em vista a inibição competitiva entre $P$ e $Z n$, segundo ROBSON \& PITMAN (1983) altas doses de $\mathrm{P}$ aplicadas em solos com teores baixos de $\mathrm{Zn}$ podem induzir à deficiência de Zn.

Além da classificação dos teores dos minerais isoladamente, também se realizou a análise de correlação (Tabela 3), onde foi possível observar que apenas as relações entre $\mathrm{P} / \mathrm{Ca}, \mathrm{P} / \mathrm{Cu}, \mathrm{Ca} / \mathrm{Mg}$ e $\mathrm{Ca} / \mathrm{B}$ apresentaram correlação moderada, as demais correlações foram classificadas como fracas ou nulas.

A correlação moderada $\mathrm{P} / \mathrm{Ca}$ pode ser resultado do manejo de implantação dos pomares. Nos pomares onde houve correção da acidez do solo (calagem) há uma maior disponibilidade de Ca e de $\mathrm{P}$ pela elevação do $\mathrm{pH}$, e o contrário é verdadeiro, solos sem correção da acidez tem menos Ca disponível e consequentemente menor disponibilidade de $\mathrm{P}$, ocasionando uma correlação positiva moderada entre $\mathrm{P} e$ Ca. 
Tabela 3. Correlação linear de Pearson entre os nutrientes em folhas de pessegueiros.

Table 3. Pearson's correlation coefficient between nutrients in peach leaves.

\begin{tabular}{cccccccccc}
\hline & $\mathrm{N}$ & $\mathrm{P}$ & $\mathrm{K}$ & $\mathrm{Ca}$ & $\mathrm{Mg}$ & $\mathrm{B}$ & $\mathrm{Cu}$ & $\mathrm{Fe}$ & $\mathrm{Mn}$ \\
\hline $\mathrm{P}$ & -0.058 & & & & & & & & \\
$\mathrm{~K}$ & 0.007 & $0.321^{*}$ & & & & & & & \\
$\mathrm{Ca}$ & -0.089 & $0.541^{* *}$ & 0.274 & & & & & & \\
$\mathrm{Mg}$ & $-0.235^{*}$ & $0.377^{*}$ & -0.127 & $0.575^{\star *}$ & & & & & \\
$\mathrm{~B}$ & -0.019 & $0.279^{*}$ & 0.110 & $0.402^{\star *}$ & 0.064 & & & & \\
$\mathrm{Cu}$ & 0.091 & $0.489^{* *}$ & 0.152 & $0.333^{*}$ & 0.150 & $0.225^{*}$ & & & \\
$\mathrm{Fe}$ & -0.002 & -0.038 & -0.085 & -0.144 & -0.190 & $-0.217^{*}$ & -0.181 & & \\
$\mathrm{Mn}$ & -0.014 & $-0.209^{*}$ & -0.070 & $-0.239^{*}$ & $-0.245^{*}$ & $-0.243^{*}$ & -0.001 & 0.000 & \\
$\mathrm{Zn}$ & 0.162 & 0.106 & $0.231^{*}$ & 0.140 & -0.009 & 0.144 & -0.029 & 0.074 & $0.292^{*}$
\end{tabular}

Valores seguidos por * = Correlação fraca $(0,20$ a 0,39$) ;{ }^{* *}=$ Correlação moderada $(0,40$ a 0,69$)$, sem asterisco = Correlação muito fraca (nula).

Observou-se correlação positiva moderada entre P/Cu. RIETRA et al. (2017) relatam que a relação entre os dois nutrientes pode ser sinérgica ou antagônica, dependendo do tipo de solo e espécie. Neste trabalho, pode-se atribuir esta correlação a alta variabilidade nutricional entre as amostras, levando a uma correlação positiva moderada. Esta hipótese pode ser estendida a relação $\mathrm{Ca} / \mathrm{Mg}$, considerando o fato de ser uma relação antagônica, pode-se afirmar que, neste caso, pomares nutricionalmente equilibrados tiveram maiores teores dos dois nutrientes e o contrário é verdadeiro. Além disso, o calcário disponível na região é o dolomítico, que tem altas concentrações de $\mathrm{Mg}$, fazendo com que haja uma elevação significativa dos teores de $\mathrm{Mg}$ através da calagem.

O Ca influencia a disponibilidade de B e sua utilização pelas plantas (KANWAL et al. 2008) e combinados, apresentam importância para a germinação do grão de pólen e crescimento do tubo polínico, funções essenciais na fecundação das flores, e taxa de pegamento dos frutos (LEITE et al. 2011). A correlação positiva entre $\mathrm{Ca}$ e $\mathrm{B}$ pode ser resultado da fertilidade do solo dos pomares, há grande variação das condições químicas dos solos, tipos de manejo e perfil do produtor, logo, em pomares menos produtivos, há menores teores destes dois nutrientes e pomares mais produtivos tem níveis maiores de $\mathrm{Ca}$ e B, levando a uma correlação positiva.

\section{CONCLUSÃO}

Há um grande desbalanço nutricional da cultura do pessegueiro na região produtora de Pelotas, RS, caracterizada pelo excesso de alguns nutrientes, como K e deficiência de outros como N, Cu, Fe e Zn.

Os teores de $\mathrm{N}$ encontrados indicam a necessidade de revisão dos valores de referência para a região, indicando também a necessidade de estudos complementares a fim de definir o nível crítico de $\mathrm{N}$ nas folhas nesta região.

A grande variação entre as amostras dos teores de minerais nas folhas indica a falta de acompanhamento do estado nutricional dos pomares de pessegueiro, principalmente via análise foliar.

A baixa correlação entre alguns nutrientes comumente correlacionados indica grande variabilidade entre os teores e amostras e desbalanço nutricional.

\section{AGRADECIMENTOS}

Ao Conselho Nacional de Desenvolvimento Científico e Tecnológico (CNPq), Universidade Federal de Pelotas (UFPel) e Embrapa Clima Temperado.

\section{REFERÊNCIAS}

AGROMET. 2018. Laboratório de Agrometeorologia, Embrapa Clima Temperado. Temperatura Média $\left({ }^{\circ} \mathrm{C}\right)$ Mensal e Precipitação Média Mensal (mm), Período 2018. Disponível em: http://agromet.cpact.embrapa.br/. Acesso em: 22 mai. 2020.

ALVARES CA et al. 2013. Köppen's climate classification map for Brazil. Meteorologische Zeitschrift 22: 711-728.

CARMO CAFS et al. 2000. Métodos de análise de tecidos vegetais utilizados na Embrapa Solos. Rio de Janeiro: Embrapa Solos. 41p. (Circular Técnica). 
CQFS-RS/SC. 2016. Comissão de Química e Fertilidade do Solo. Manual de adubação e calagem para os estados do Rio Grande do Sul e de Santa Catarina. 10.ed. Porto Alegre: SBCS. 376p.

FERREIRA LV et al. 2018. Adubação nitrogenada em ciclos consecutivos e seu impacto no manejo adensado de pessegueiro. Pesquisa Agropecuária Brasileira 53: 172-181.

FREIRE CJ da S \& MAGNANI M. 2014. Adubação e correção do solo. In: RASEIRA MCB et al. (Ed.). Pessegueiro. Brasília: Embrapa. p. 259-281.

HOCKING B et al. 2016. Fruit calcium: transport and physiology. Frontiers in Plant Science 7: 569.

IBGE. 2018. Instituto Brasileiro de Geografia e Estatística. Produção agrícola municipal. Rio de Janeiro. Disponível em: http://www.sidra.ibge.gov.br/bda/tabela/protabl.asp?c=1613\&z=\&o=24\&i=P. Acesso em: 09 mar. 2019.

JOHNSON RS. 2008. Nutrient and Water Requirements of Peach Trees. In. LAYNE DR \& BASSI D. The Peach: Botany, Production and Uses. Wallingford: CABI. 615p.

JOHNSON RS \& URIU K. 1989. Mineral nutrition. In: LARUE JH. Peaches, plums, and nectarines: Growing and handling for fresh market. Oakland: University of California. p. 68-81.

KANWAL $S$ et al. 2008. Critical ratio of calcium and boron in maize shoot for optimum growth. Journal of Plant Nutrition. 31: 1535-1542.

LEITE RFC et al. 2011. Rendimento e qualidade de sementes de arroz irrigado em função da adubação com boro. Revista Brasileira de Sementes 33: 785-791.

MAYER NA et al. 2019. Pêssego, nectarina e ameixa: o produtor pergunta, a Embrapa responde. Brasília: Embrapa. 290p.

MAYER NA et al. 2016. Adensamento de plantio em pessegueiros 'Chimarrita'. Revista de Ciências Agroveterinárias 15: 50-59.

MAYER NA et al. 2015. A morte precoce do pessegueiro associada à fertilidade do solo. Revista Brasileira de Fruticultura 37: 773-787.

NAVA G \& CARVALHO FLC. 2019. Calagem, adubação e nutrição. In MAYER NA et al. Pêssego, nectarina e ameixa: o produtor pergunta, a Embrapa responde. Brasília: Embrapa. p.115-125.

NAVROSKI R et al. 2019a. Response of 'Sensação' peach trees to phosphate fertilization. Semina: Ciências Agrárias 40: 3345-3382.

NAVROSKI R et al. 2019b. Adubação fosfatada em pessegueiros: É preciso aplicar fósforo todos os anos? Jornal da Fruta 338: 7.

RIETRA RP et al. 2017. Effects of nutrient antagonism and synergism on yield and fertilizer use efficiency. Communications in soil science and plant analysis 48: 1895-1920.

ROBSON AD \& PITMAN MG. 1983. Interactions between nutrients in higher plants. In: LAUCHILI A \& BIELESKI RL. (Ed.). Inorganic plant nutrition. Berlin: Springer-Verlag. p.147-180.

ROMBOLÀ AD et al. 2012. Nutrição e manejo do solo em fruteiras de caroço em regiões de clima temperado. Semina: Ciências Agrárias 33: 639-654.

SEVERO PS. 2017. Os pêssegos não caem do céu: relações de trabalho na agricultura familiar no município de Pelotas/RS. Tese (Doutorado em Agronomia). Pelotas: UFPEL. 148p.

SHEAR CB \& FAUST M. 1980. Nutritional Ranges in Deciduous Tree Fruits and Nuts. Horticultural Reviews 2: $142-163$. SHIKAMURA SE. 2006. Correlação. CE003 - Estatística II. Curitiba: UFPR. p.71-78.

TAGLIAVINI M et al. 2000. Ripartizione degli elementi minerali nei frutti degli alberi decidui. Rivista di Frutticoltura 62: 83-87.

TAYLOR KC. 2009. Cultural management of the bearing peach orchard. Georgia: The University of Georgia. 4p. Circular 879. 